Research Article

\title{
Imperfect Fluid Generalized Robertson Walker Spacetime Admitting Ricci-Yamabe Metric
}

\author{
Ali H. Alkhaldi $\mathbb{D}^{1},{ }^{1}$ Mohd Danish Siddiqi $\mathbb{D D}^{2}{ }^{2}$ Meraj Ali Khan $\left(\mathbb{D},{ }^{3}\right.$ \\ and Lamia Saeed Alqahtani $\mathbb{1}^{4}$ \\ ${ }^{1}$ Department of Mathematics, College of Science, King Khalid University, P.O. Box 9004, Abha, Saudi Arabia \\ ${ }^{2}$ Department of Mathematics, College of Science, Jazan University, Jazan, Saudi Arabia \\ ${ }^{3}$ Department of Mathematics, College of Science, University of Tabuk University, Tabuk, Saudi Arabia \\ ${ }^{4}$ Department of Mathematics, Faculty of Science, King Abdulaziz University, Jeddah 21589, Saudi Arabia
}

Correspondence should be addressed to Meraj Ali Khan; meraj79@gmail.com

Received 7 July 2021; Revised 2 November 2021; Accepted 2 December 2021; Published 16 December 2021

Academic Editor: Remi Léandre

Copyright $\odot 2021$ Ali H. Alkhaldi et al. This is an open access article distributed under the Creative Commons Attribution License, which permits unrestricted use, distribution, and reproduction in any medium, provided the original work is properly cited.

In the present paper, we investigate the nature of Ricci-Yamabe soliton on an imperfect fluid generalized Robertson-Walker spacetime with a torse-forming vector field $\xi$. Furthermore, if the potential vector field $\xi$ of the Ricci-Yamabe soliton is of the gradient type, the Laplace-Poisson equation is derived. Also, we explore the harmonic aspects of $\eta$-Ricci-Yamabe soliton on an imperfect fluid GRW spacetime with a harmonic potential function $\psi$. Finally, we examine necessary and sufficient conditions for a 1 -form $\eta$, which is the $g$-dual of the vector field $\xi$ on imperfect fluid GRW spacetime to be a solution of the SchrödingerRicci equation.

\section{Introduction}

Symmetry is a beautiful property of the universe. It is also one of the fundamental concepts that can describe the laws of nature such as from general relativity to other physical theories. In 1915, Albert Einstein introduced the theory, namely, "General Relativity of gravity" (GR). In GR, the field of gravity with its source is the spacetime curvature and energymomentum tensor, respectively. Einstein equations which explain spacetime curvature evolution lead to current particle physics, equations in nuclear physics [1], astrophysics [2], and plasma physics [3]. To understand the general theory of relativity, we study the model of relativistic fluids from the view of differential geometry. The general theory of relativity is based on the concept that spacetime is a curved manifold.

According to J. A. Wheeler, "Matter tells spacetime how to bend and spacetime returns the complement by telling matter how to move."
The spacetime of GR and cosmology is modeled as a connected 4-dimension Lorentzian manifold considered as a specific subclass of pseudo-Riemannian manifolds among Lorentzian metric $g$, where its signatures $(-,+,+,+)$ play an important role in GR. The geometry of Lorentzian manifold is connected to the nature of vectors of manifold. As a result, Lorentzian manifold is the best model to investigate GR.

Alias et al. [4] presented the concept of generalized Robertson-Walker spacetime (GRW, in short) that generalizes the Robertson-Walker (RW) spacetime which is a direct application of warped product manifolds.

Definition 1 [4]. A Lorentzian manifold $M$ of dimension $n$ $\geq 3$ is said to be a GRW spacetime if it is the warped product $M=I \times{ }_{f} M^{*}$ with an open interval $\left(I,-d t^{2}\right)$ of $\mathbb{R}$ and a Riemannian manifold $M^{*}$ with warping function $f: I \longrightarrow \mathbb{R}^{+}$. 
Definition 2 [5]. An $n$-dimensional Lorentzian manifold is named GRW spacetime if the metric takes the local form

$$
d s^{2}=-(d t)^{2}+r(t)^{2} g_{i j}^{*} d x^{i} d x^{j}
$$

where $g^{*}=g_{i j}^{*}\left(x^{k}\right)$ are functions of $x^{k}$ only $(i, j, k=2,3, \cdots, n)$ and $r$ is the function of $t$ only. If $g_{i j}^{*}$ has dimension 3 and has constant curvature, the space is a Robertson-Walker spacetime.

Definition 3 [6]. A nonflat semi-Riemannian manifold of dimension $n>2$ is known as pseudo quasi Einstein manifolds, if its nonzero Ricci tensor satisfies

$$
S(U, V)=a g(U, V)+b \eta(U) \eta(V)+c D(U, V),
$$

where $a, b$, and $c$ are nonzero scalars, $\eta$ is a nonzero 1 -form associated with the unit timelike vector field $\xi$ defined by $\eta$ $(U)=g(U, \xi)$, and $D$ is a symmetric tensor with zero trace defined as $D(U, \xi)=0$.

A GRW spacetime with dimension $n$ is the $n$-dimensional Lorentzian manifold $M$. According to Sanchez [7], the GRW spacetime has applications in the homogeneous spacetime with an isotropic radiation. O'Neil [8] in his book listed that an RW spacetime is the imperfect fluid spacetime. If the dimension of GRW spacetime is $n=4$, then it becomes a perfect fluid spacetime if and only if it is an RW spacetime [5].

In geometry, symmetry is used to describe the distribution of physical objects, particularly in relation to the geometry of spacetime. In most cases, the metric of symmetry simplifies the solution in various studies. More specifically, Ricci curvature has several applications in GR, for instance, in the solution of Einstein field equations. Solitons are one of the most important symmetry patterns, which are related to the geometrical flow of spacetime geometry. For instance, Ricci flow and Yamabe flow in which vital terms have been used to understand energy and entropy in GR. Moreover, various studies in GR show that Ricci soliton and Yamabe soliton are focused because their curvatures preserve selfsimilarity.

In [9], Ali and Ahsan studied the symmetries of space time manifold via Ricci solitons. However, Blaga [10] discussed geometrical aspects for the perfect fluid spacetime by using Einstein solitons and Ricci solitons. In addition, Venkatesha and Aruna [11] used Ricci solitons in the study of perfect fluid spacetime admitting the potential vector field. Many researchers have performed extensive research on solitons with spacetimes by using different methods (for more details, see $[10,12-14])$.

As a result, we concentrate on the geometry of an imperfect fluid spacetime admitting the Ricci-Yamabe soliton and an eta-Ricci-Yamabe soliton to continue the work initiated in the past studies. We develop a new notion of RicciYamabe soliton and its extension $\eta$-Ricci-Yamabe soliton with the help of Ricci-Yamabe maps studied by Güler and Crasmareanu [15].

\section{Development of Ricci-Yamabe Solitons}

In 1988, Hamilton [16] first time introduced the concept of Ricci flow and Yamabe flow simultaneously. Ricci soliton and Yamabe soliton appear in the limiting case of the Ricci flow and Yamabe flow, respectively. If dimension of Yamabe soliton is $n=2$, then it turns to Ricci soliton, but when $n>2$, Yamabe and Ricci solitons are not identical in general, because Yamabe soliton keeps the conformal class of the metric while Ricci soliton does not.

Over the past twenty years, many geometers and physicists have been fascinated by the theory of geometric flow such as Ricci flow and Yamabe flow. A singularity study is a part of the solution where the metric develops through dilation and diffeomorphism, soliton solution is a common term for this type of solution.

In 2019, Güler and Crasmareanu [15] introduced the study of a new geometric flow, namely, Ricci-Yamabe map, which is a scalar combination of Ricci and Yamabe flow; this is also called Ricci-Yamabe flow of the type $(\alpha, \beta)$. The RicciYamabe flow is the evolution of metrics at the Riemannian or semi-Riemannian manifold defined as [15].

$$
\frac{\partial}{\partial t} g(t)=-2 \alpha S(t)+\beta R(t) g(t), g_{0}=g(0), t \in(a, b)
$$

Due to the involvement of scalars $\alpha$ and $\beta$, the RicciYamabe flow may be a Riemannian or semi-Riemannian or singular Riemannian flow; multiple options like this can be advantageous in some geometrical or physical models, for example, relativistic theories. As a result, the Ricci-Yamabe soliton appears as the limit of soliton for the Ricci-Yamabe flow; this is a strong motivation for us to develop the concept of Ricci-Yamabe solitons. In [17], Catino and Mazzieri presented an interpolation solitons between Ricci and Yamabe soliton, where the Ricci-Bourguignon soliton corresponds to Ricci-Bourguignon flow, but it depends on a scalar.

A soliton of Ricci-Yamabe flow which moves just by one parameter group of diffeomorphism and scaling is named Ricci-Yamabe soliton. To be precise, the Ricci-Yamabe soliton at Riemannian manifold $(M, g)$ is the structure $(g, V$, $\lambda, \alpha, \beta)$ satisfying

$$
\mathscr{L}_{V} g+2 \alpha S+(2 \lambda-\beta R) g=0
$$

where $S$ is Ricci tensor, $R$ is scalar curvature, $\mathscr{L}_{V}$ is Liederivative along the vector field $V$, and $\alpha$ and $\beta$ are constant. The $(M, g)$ is called Ricci-Yamabe shrinker, Ricci-Yamabe expander, or Ricci-Yamabe steady soliton, depending on whether $\lambda>0, \lambda<0$, or $\lambda=0$, respectively. Therefore, equation (4) is called Ricci-Yamabe soliton of $(\alpha, \beta)$-type, which is a generalization of Ricci and Yamabe solitons. We note that Ricci-Yamabe solitons of type $(\alpha, 0)$ and $(0, \beta)$-type are $\alpha$-Ricci soliton and $\beta$-Yamabe soliton, respectively.

A notion for $\eta$-Ricci soliton defined as in [18] is an advance extension for Ricci soliton. Therefore, we can define a new notion in an analogous fashion by perturbing the equation (4) that defines the type of soliton using the multiplication of the certain $(0,2)$-tensor field $\eta \otimes \eta$; a slightly 
more general notion is obtained, specifically $\eta$-Ricci-Yamabe soliton of type $(\alpha, \beta)$ defined as:

$$
\mathscr{L}_{V} g+2 \alpha S+(2 \lambda-\beta R) g+2 \mu \eta \otimes \eta=0 .
$$

Again, let us remark that $\eta$-Ricci-Yamabe solitons of type $(\alpha, 0)$ or $(1,0)$ and $(0, \beta)$ or $(0,1)$-type are $\alpha-\eta$-Ricci soliton (or $\eta$-Ricci soliton) and $\beta-\eta$-Yamabe soliton (or $\eta$ Yamabe soliton), respectively; for more details about these particular cases, one can follow ([19-24]).

Example 4. Let us consider the case of Einstein soliton, that is generating self-similar solutions of Einstein flow [17], which is given by

$$
\frac{\partial}{\partial t} g(t)=-2\left(S-\frac{R}{2} g\right) .
$$

Therefore, an Einstein soliton appears as the solution limit of the Einstein flow, which is governed by the following formula

$$
\frac{1}{2} \mathscr{L}_{V} g+S+\left(\lambda-\frac{R}{2}\right) g=0 .
$$

In this case, comparing equation (7) with (4), we have $\alpha=1$ and $\beta=1$, i.e., it is a type of $(1,1)$-Ricci-Yamabe soliton.

Example 5. Let us consider the conformal Ricci flow equation which was studied in [25], which is characterized by the following tensorial equation

$$
\frac{\partial g}{\partial t}=-2 S-\left(p+\frac{2}{n}\right) g
$$

where $r(g)=-1, t \in(a, b)$, a time interval including $g(0), p$ is a nondynamical scalar field (time dependent scalar field), $r$ $(g)$ is the scalar curvature of the manifold, and $n$ is the dimension of the manifold $M$. The notion of conformal Ricci soliton is governed by the following equation

$$
\mathscr{L}_{V} g+2 S+\left[2 \lambda-\left(p+\frac{2}{n}\right)\right] g=0
$$

On comparing equation (9) with (4), we have $\alpha=1$ and $\beta=-1$, i.e., it is a type $(1,-1)$ Ricci-Yamabe soliton.

2.1. Geometrical and Physical Effects of Ricci-Yamabe Solitons. Geometry of Ricci-Yamabe solitons can develop a bridge between the curvature inheritance symmetry for the imperfect fluid spacetime (semi-Riemannian manifold) and class of the Ricci-Yamabe solitons. For this, three mathematical forms are constructed for semiconformally flat Ricci-Yamabe soliton manifolds. To investigate the kinematic and dynamic properties of spacetime in order to apply relativity, the physical model is presented for the three classes, namely, expanding, steady, and shrinking of perfect fluid solution for RicciYamabe soliton spacetime.

To deal with these specific classes of Ricci-Yamabe solitons, specifically shrinking $(\lambda<0)$, where it happens on the maximal time interval $-\infty<t<b$ and $b<\infty$, steady $(\lambda=0)$ where it happens for every time or expanding $(\lambda>0)$ that occurs at maximal time interval $a<t<\infty$, where $a>-\infty$ [26]. These classes yield examples of ancient, eternal, and immortal solution, in the same order. Additionally, shrinking or expanding Ricci-Yamabe solitons are linked to Einstein gravity coupled to a free mass less scalar field with nonzero cosmological constant.

\section{Preliminaries}

The energy-momentum tensor is used as a basic tool of the spacetime, assuming the fluid which have density, pressure, dynamical, and kinematic quantities such as velocity, acceleration, vorticity, shear, and expansion [27]. If the viscosity terms are non-zero, the fluid is named an imperfect fluid [28]. Imperfect fluid spacetime can give an adequate details for cosmological models beyond the standard model like perfect fluid spacetimes. The complete idea of the nature deals with the behavior of the perfect fluid and imperfect fluid spacetime in standard cosmological models. The Brans-Dicke-like field of scalar-tensor gravity is identified as the imperfect fluid that is described by an effective Einstein equation. In Einstein's theory, the effective imperfect fluid explanation is presented for the canonical; GRW spaces applied at Friedmannian cosmology [29].

Now, we should state the following definitions, which will come in handy in the next parts.

Definition 6 [30]. A vector field $\gamma_{j}$ on the semi-Riemannian manifold is called torse-forming vector field in case

$$
\nabla_{k} \gamma_{j}=\omega_{k} \gamma_{j}+\varphi g_{k j}
$$

where $\varphi$ is the scalar function and $\omega_{k}$ is a nonvanishing 1 -form.

Clearly, the unit timelike torse-forming vector field $u_{i}$ on the semi-Riemannain manifold $M$ is given as:

$$
\nabla_{k} u_{j}=\varphi\left(g_{k j}+u_{k} u_{j}\right)
$$

In addition, we have some significant results based on GRW spacetime.

Theorem 7 [22]. A Lorentzian manifold $M$ with $\operatorname{dim}(M) \geq 3$ is a GRW spacetime if and only if it admits a timelike concircular vector field.

In 2017, Mantica and Molinari [5] have established the necessary and sufficient conditions for a Lorentzian manifold to admit the unit timelike torse-forming vector field to be GRW spacetime, that is also an eigenvector of Ricci tensor. 
In a spacetime with Lorentzian metric $g_{i j}$, the stressenergy-momentum tensor $T$ with heat flow for an imperfect fluid GRW spacetime can be written as $[8,29,31]$

$$
T(U, V)=p g(U, V)+(\sigma+p) \eta(U) \eta(V)+P(U, V)
$$

where $\sigma$ and $p$ define the energy density and isotropic pressure, respectively, and $P$ defines the tensor of isotropic pressure for the viscous fluid [28].

The Einstein's gravitational equation which controls the fluid motion is given by the following relation [8]

$$
S(U, V)+\left(\lambda-\frac{R}{2}\right) g(U, V)=\kappa T(U, V)
$$

for all $U, V \in \chi(M)$, where $\lambda$ is the cosmological constant, $\kappa$ is the gravitational constant (that is taken $8 \pi G, G$ represents the universal gravitational constant), $S$ is Ricci tensor, and $R$ is scalar curvature of $g$. Basically, the universe is filled with the mysterious components, and these are called dark energy $\mathrm{DE}$ and dark matter DM; they are considered to be the main reason for the accelerated expansion of the universe and balance the mass-energy ratio.

Also, using equation (12) as well as (13) for an imperfect fluid GRW spacetime, we get

$$
S(U, V)=a g(U, V)+b \eta(U) \eta(V)+c P(U, V)
$$

where $a=(-\lambda+R / 2+\kappa p), b=\kappa(\sigma+p)$, and $c=\kappa$. Thus, in the light of (14) and (2), we can state the following result.

Theorem 8. An imperfect fluid GRW spacetime with stress energy tensor described by (12), obeying Einstein's field equation with cosmological constant, is a pseudo quasi-Einstein GRW spacetime.

\section{Imperfect Fluid Generalized Robertson- Walker Spacetime}

Here, we discuss the basic concepts of GRW spacetime.

Suppose $\left(M^{4}, g\right)$ is the relativistic imperfect fluid GRW spacetime satisfying (14), then by (14) and the assumption that $g(\xi, \xi)=-1$, we have

$$
R=4 \lambda-\kappa[3 p-\sigma+J]
$$

where $J=\operatorname{trace}(P)$. Now, we can deduce that

$$
S(U, V)=\left(\lambda-\frac{\kappa}{2}[p-\sigma+2 J]\right) g(U, V)+\kappa(\sigma+p) \eta(U) \eta(V)+\kappa P(U, V),
$$

$$
Q U=a U+b \eta(U) \xi
$$

where $a=(\lambda-\kappa / 2(p-\sigma+2 J))$ and $b=\kappa(1+\sigma+p)$. Also

$$
S(\xi, \xi)=\frac{\kappa}{2}[3 p+\sigma+2(J+I)]-\lambda,
$$

where $I=P(\xi, \xi)$.

It is noted that, in a GRW spacetime, the velocity field of a perfect fluid or an imperfect fluid described by the stressenergy tensor (12) is a torse-forming and proportional to Chen's vector which is defined in $[5,29]$.

Motivated by the results of (see $[5,22,29])$ together with the above facts and Definition 6, regarding to global expressions, the next theorem for an imperfect GRW spacetime is stated. [32]:

Theorem 9. On an imperfect fluid GRW spacetime with a unit timelike torse-forming vector field $\xi$, the following relations hold

$$
\begin{gathered}
\eta\left(\nabla_{U} \xi\right)=0, \nabla_{\xi} \xi=0, \\
\left(\nabla_{U} \eta\right)(V)=\varphi[g(U, V)+\eta(U) \eta(V)], \\
R(U, V) \xi=\varphi[\eta(V) U-\eta(U) V], \\
R(\xi, U) V=\varphi[\eta(V) U-g(U, V) \xi], \\
\eta(R(U, V) W=)=\varphi[\eta(U) g(V, W)-\eta(V) g(Y, W)], \\
\left(\mathscr{L}_{\xi} g\right)(U, V)=2 \varphi[g(U, V)+\eta(U) \eta(V)], \\
S(U, \xi)=-3 \varphi \eta(U) .
\end{gathered}
$$

Proof. To compute $\left(\nabla_{U} \eta\right)(V)=U\left(\eta(V)-\eta\left(\nabla_{U} V\right)\right)=U(g($ $\left.V, \xi)-g\left(\nabla_{U} V, \xi\right)\right)=g\left(V, \nabla_{U} \xi\right)=\varphi[g(U, V)+\eta(U) \eta(V)]$. In particular $\left(\nabla_{\xi} \eta\right)(V)=0$, equation (19) is given as (11).

Substituting the term of $\nabla_{U} \xi$ from (11) into $R(U, V) \xi$ $=\nabla_{U} \nabla_{V} \xi-\nabla_{V} \nabla_{U} \xi-\nabla_{[U, V]} \xi$ and by direct calculations, we find the relation (21), (22), and (25).

Here, the Lie derivative of $g$ with respect to $\xi$ is followed by straight forward computation, which is (24).

\section{Geometrical Characteristics of Imperfect Fluid GRW Spacetime}

In this section, we discuss the properties of a new curvature tenor called semiconformal curvature tensor and its relationship with imperfect fluid GRW spacetime.

In 2017, Kim [33] presented curvature like-tensor field that remains invariant with respect to conharmonic transformation. The new tensor is named as semiconformal curvature tensor denoted by $H$. However, for the semiRiemannian manifold $M$ with metric $g$, the tensor $H$ is given by the following formula [34]

$$
H(U, V) W=-(n-2) \delta C(U, V) W+[\varepsilon+(n-2) \delta] L(U, V) W \text {, }
$$

provided the constants $\varepsilon$ and $\delta$ are not simultaneously zero, 
where $C$ and $L$ are conformal curvature tensor as well as conharmonic curvature tensor in the same order.

Now, an imperfect fluid GRW spacetime with a unit timelike torse-forming vector field of dimension 4 named semiconformally flat imperfect fluid GRW spacetime; if the semiconformal curvature tensor $H$ vanishes then, we get the following information by equation (26).

Suppose $\left(M^{4}, g\right)$ is the semiconformally flat imperfect fluid GRW spacetime with a unit timelike torse-forming vector field $\xi$.

As $H(U, V) W=0$, it gives div $H=0$, where div denotes the divergence of a vector. Now, (26) leads to

$$
\left(\nabla_{U} S\right)(V, W)-\left(\nabla_{V} S\right)(U, W)=\frac{\delta}{3 \varepsilon}[U(R) g(V, W)-V(R) g(U, W)],
$$

or

$$
g\left(\left(\nabla_{U} Q\right) V-\left(\nabla_{V} Q\right) U, W\right)=\frac{\delta}{3 \varepsilon}[U(R) g(V, W)-V(R) g(U, W)] .
$$

Since, in light of (15), scalar curvature $R$ is constant, and from (17), equation (28) leads to

$0=\left(\nabla_{U} Q\right)-\left(\nabla_{V} Q\right) U=b\left[\left(\nabla_{U} \eta\right)(V) \xi+\eta(V) \nabla_{U} \xi-\left(\nabla_{V} \eta\right)(U) \xi-\eta(V) \nabla_{U} \xi\right]$.

Then, between (11) and (20), we find

$$
b[\eta(V) U-\eta(U) V]=0,
$$

that gives $b=0$ and leads to $p=-(\sigma+1)$; the energymomentum tensor is Lorentz-invariant, and in such a case, we discuss about vacuum.

By (17), it is easy to conclude $Q U=a U$. Therefore, $H=0$ , and it leads to

$R(U, V) W=\frac{2 \delta(\lambda+\kappa[1 / 2+\sigma+J])}{3 \varepsilon}[g(V, W) U-g(U, W) V]$,

which means $\left(M^{4}, g\right)$ is of constant curvature $2 \delta(\lambda+\kappa[1 / 2$ $+\sigma+J]) / 3 \varepsilon$; by the applications of (31), we have the following result:

Theorem 10. If imperfect fluid GRW spacetime with a unit timelike torse-forming vector field $\xi$ and constant scalar curvature $R$ is semiconformally flat, then the stress-energy tensor is Lorentz-invariant and is of constant curvature 2 $\delta(\lambda+\kappa[1 / 2+\sigma+J]) / 3 \varepsilon$.

The pseudo-Riameannian manifold is called a quasiconstant curvature, if the curvature tensor of the type $(0,4)$ satisfies

$$
\begin{aligned}
R\left(U, V, W, W^{\prime}\right)= & m\left[g(V, W) g\left(U, W^{\prime}\right)-g(U, W) g\left(V, W^{\prime}\right)\right] \\
& +n\left[g\left(U, W^{\prime}\right) \eta(V) \eta(W)-g(U, W) \eta(V) \eta\left(W^{\prime}\right)\right. \\
& \left.+g(V, W) \eta(U) \eta\left(W^{\prime}\right)-g\left(V, W^{\prime}\right) \eta(U) \eta\left(W^{\prime}\right)\right],
\end{aligned}
$$

where $m$ and $n$ are scalars and $\eta$ is nonzero 1 -form that is $g(U, Z)=\eta(U)$, for any unit vector fields $U, Z$. The concept of the manifold with quasi-constant curvature was presented by Yano [35].

On using equation (31) in (32), we get

Corollary 11. A semiconformally flat imperfect fluid GRW spacetime with a unit timelike torse-forming vector field $\xi$ is of quasi-constant curvature with $m=2 \delta(\lambda+\kappa[1 / 2+\sigma+J]) /$ $3 \varepsilon$ and $n=0$.

It is well known that the manifold of constant curvature is Einstein manifold; now by the application of Theorem 10, we state the following theorem:

Theorem 12. The semiconformally flat imperfect fluid GRW spacetime with a unit timelike torse-forming vector field $\xi$ is an Einstein.

A pseudo-Riemannian manifold $(M, g)$ is called semisymmetric and Ricci semisymmetric if $(M, g)$ hold the conditions $R(U, V) \cdot R=0$ and $R(U, V) \cdot S=0$, in the same order. The restriction $R(U, V) \cdot R=0$ gives $R(U, V) \cdot S=0$, but the other does not true in general.

Now, we prove the next theorem;

Theorem 13. A semiconformally flat imperfect fluid GRW spacetime with a unit timelike torse-forming vector field $\xi$ is semisymmetric and Ricci semisymmetric.

Proof. From equation (31), it is clear that $R(U, V) \cdot R=0$, and this gives $R(U, V) \cdot S=0 . \square$

\section{Ricci-Yamabe Soliton Structure in an Imperfect Fluid GRW Spacetime}

In this section, we deal with Ricci-Yamabe soliton of type $(\alpha, \beta)$ in an imperfect fluid GRW spacetime whose unit timelike velocity vector field $\xi$ is torse-forming.

Now, taking $V=\xi$, equation (4) becomes

$$
\mathscr{L}_{\xi} g(U, V)+2 \alpha S(U, V)+(2 \Omega-\beta R) g(U, V)=0
$$

where $R$ is scalar curvature. Now using (24), we find

$$
\alpha S(U, V)+\left(\Omega-\frac{\beta R}{2}+\varphi\right) g(U, V)+\varphi \eta(U) \eta(V)=0
$$


Putting $U=V=\xi$ in (34) and using (18), we get

$$
\Omega=\left\{\frac{\beta R}{2}\right\}+\alpha\left[\frac{\kappa}{2}\{\sigma+3 p+2(J+I)\}-\lambda\right] .
$$

Hence, we give the following:

Theorem 14. Let an imperfect fluid GRW spacetime with a unit timelike torse-forming vector field $\xi$ admitting a RicciYamabe soliton $(g, \xi, \Omega)$ of type $(\alpha, \beta)$, then the RicciYamabe soliton is expanding.

Corollary 15. If an imperfect fluid GRW spacetime with a unit timelike torse-forming vector field $\xi$ admitting the Ricci-Yamabe soliton $(g, \xi, \Omega)$ of type $(0, \beta)$, then the $\beta$ Yamabe solitons is expanding.

Corollary 16. If an imperfect fluid GRW spacetime with a unit timelike torse-forming vector field $\xi$ admitting a RicciYamabe soliton $(g, \xi, \Omega)$ of type $(\alpha, 0)$, then the RicciYamabe soliton expands, steady, and shrinks according as
(i) $(\kappa / 2)\{\sigma+3 p+2(J+I)\}>\lambda$
(ii) $(\kappa / 2)\{\sigma+3 p+2(J+I)\}=\lambda$ and
(iii) $(\kappa / 2)\{\sigma+3 p+2(J+I)\}<\lambda$, respectively

Moreover, if $J=I=0$ for the perfect fluid GRW spacetime, then we turn up the following:

Theorem 17. If a perfect fluid GRW spacetime with a unit timelike torse-forming vector field $\xi$ admitting a RicciYamabe soliton $(g, \xi, \Omega)$ of type $(\alpha, \beta)$, then the RicciYamabe soliton is expanding.

Remark 18. According to the above corollaries (15) and (16), we can easily obtain the similar results for perfect fluid GRW spacetime.

\section{7. $\eta$-Ricci-Yamabe Soliton in an Imperfect Fluid GRW Spacetime}

Consider the equation

$$
\mathscr{L}_{\xi} g+2 \alpha S+(2 \Omega-\beta R) g+2 \mu \eta \otimes \eta=0
$$

where $g$ is a Lorentzian metric, $S$ is a Ricci curvature, $\xi$ is the vector field, $\eta$ is the 1 -form, and $\Omega$ and $\mu$ are real constant. The structure $(g, \xi, \Omega, \mu, \alpha, \beta)$ that satisfies equation (36) called $\eta$-Ricci-Yamabe soliton at $M$ [14]. In particular if $\mu$ $=0, \quad(g, \xi, \Omega)$ becomes Ricci-Yamabe soliton and it is shrinked, steady, or expanded with respect to $\Omega$ that is negative, zero, or positive, accordingly.

More specific, the Lie derivative $\mathscr{L}_{\xi} g$ gives

$$
\left(\mathscr{L}_{\xi} g\right)(U, V)=g\left(\nabla_{U} \xi, V\right)+g\left(U, \nabla_{V} \xi\right)
$$

and form (36) we obtain

$$
\begin{array}{r}
\alpha S(U, V)=-\left(\Omega-\frac{\beta R}{2}\right) g(U, V)-\mu \eta(U) \eta(V) \\
-\frac{1}{2}\left[g\left(\nabla_{U} \xi, V\right)+g\left(U, \nabla_{V} \xi\right)\right]
\end{array}
$$

for any $U, V \in \chi(M)$.

On contracting (38), we get

$$
-(\alpha-\beta) R=-4 \Omega+\mu-\operatorname{div}(\xi) .
$$

Let $\left(M^{4}, g\right)$ be a general relativistic imperfect fluid GRW -spacetime and $(g, \xi, \Omega, \mu, \alpha, \beta)$ is a $\eta$-Ricci-Yamabe soliton at $M,(16)$ and (38) lead to

$$
\begin{gathered}
\alpha\left[\lambda-\frac{\kappa(p-\sigma+2 J)}{2}+\left(\Omega-\frac{\beta R}{2}\right)\right] g(U, V) \\
+[\alpha \kappa(\sigma+p)+\mu] \eta(U) \eta(V)+\alpha \kappa P(U, V) \\
+\frac{1}{2} g\left(\nabla_{U} \xi, V\right)+g\left(U, \nabla_{V} \xi\right)=0
\end{gathered}
$$

for any $U, V \in \chi(M)$.

Considering $\left\{e_{i}\right\}_{1 \leq i \leq 4}$ is an orthonormal frame and $\xi=$ $\sum_{i=1}^{4} \xi^{i} e_{i}$, we have $\sum_{i=1}^{4} \varepsilon_{i i}\left(\xi^{i}\right)^{2}=-1$ and $\eta\left(e_{i}\right)=\varepsilon_{i i} \xi^{i}$.

By the multiplication of (40) with $\varepsilon_{i i}$, putting $U=V=e_{i}$ and summing over $i$, we have

$4 \alpha \Omega-\mu=-(\alpha+2 \alpha \beta) 4 \lambda+\kappa[(\alpha+6 \alpha \beta) p-(3 \alpha+2 \alpha \beta)(\sigma+J)]-\operatorname{div}(\xi)$.

Writing (40) with $X=Y=\xi$, which leads to

$\alpha \Omega-\mu=-(\alpha+2 \alpha+\beta) \lambda-\frac{\kappa}{2}[(\alpha-3 \alpha \beta) p+(\alpha+\alpha \beta) \sigma-(2 \alpha+\alpha \beta) J+\alpha I]$.

Therefore

$$
\left\{\begin{array}{l}
\Omega=-(1+2 \beta) \lambda+\frac{\kappa}{\alpha}\left(c_{1} p-c_{2} \sigma+c_{3} J+\frac{I}{3}\right)-\frac{\operatorname{div}(\xi)}{3 \alpha} \\
\mu=\frac{\kappa}{3}\left(3 \alpha p-c_{4} \sigma+c_{5} J-4 \alpha I\right)-\frac{\operatorname{div}(\xi)}{3}
\end{array}\right.
$$

where $c_{1}=(\alpha / 2+3 \alpha \beta / 2), c_{2}=(7 \alpha-\alpha \beta), c_{3}=(4 \alpha+\alpha \beta), c_{4}$ $=(5 \alpha+4 \alpha \beta)$, and $c_{5}=(7 \alpha+4 \alpha \beta)$. Using (43), the coming results are state.

Theorem 19. If $\left(M^{4}, g\right)$ is the general relativistic imperfect fluid GRW spacetime and let $\eta$ be the g-dual 1-form of the gradient vector field $\xi=\operatorname{grad}(\psi)$. If (36) defines an $\eta$-RicciYamabe soliton with nonvanishing $\alpha$ and $\beta$ in $M^{4}$, therefore, 
Laplace-Poisson equation insured by $\psi$ turns to

$$
\Delta(\psi)=-3\left[\mu-\frac{\kappa}{3}\left(3 \alpha p-c_{4} \sigma+c_{5} J-4 \alpha I\right)\right] .
$$

For perfect fluid GRW spacetime $J, I$ and $c_{5}$ vanishes, therefore we can turn up the following result.

Corollary 20. Let $\left(M^{4}, g\right)$ is the general relativistic perfect fluid GRW spacetime and let $\eta$ be the $g$-dual 1-form of the gradient vector field $\xi=\operatorname{grad}(\psi)$. If (36) defines an $\eta$-RicciYamabe soliton with nonvanishing $\alpha$ and $\beta$ in $M^{4}$, therefore, Laplace-Poisson equation insured by $\psi$ turns to

$$
\Delta(\psi)=-3\left[\mu-\frac{\kappa}{3}\left(3 \alpha p-c_{4} \sigma\right)\right]
$$

Example 21. An $\eta$-Ricci-Yamabe soliton $(g, \xi, \Omega, \mu, \alpha, \beta)$ at the radiation fluid is given as

$$
\left\{\begin{array}{l}
\Omega=-(1+2 \beta) \lambda+\frac{\kappa}{\alpha}\left(c_{1} p-c_{2} \sigma+c_{3} J+\frac{I}{3}\right)-\frac{\operatorname{div}(\xi)}{3 \alpha} \\
\mu=\frac{\kappa}{3}\left(3 \alpha p-c_{4} \sigma+c_{5} J-4 \alpha I\right)-\frac{\operatorname{div}(\xi)}{3}
\end{array}\right.
$$

\section{Harmonic Aspects of the $\eta$-Ricci-Yamabe Soliton on an Imperfect Fluid GRW Spacetime}

Let $\eta$ is a $g$-dual 1 -form of the given vector field $\xi$, considering $g(X, \xi)=\eta(X)$ and $g(\xi, \xi)=-1$. Then, $\xi$ is called a solution of the Schrödinger-Ricci equation if it satisfies

$$
\operatorname{div}\left(\mathscr{L}_{\xi} g\right)=0
$$

where $\mathscr{L}_{\xi} g$ is Lie derivative for the vector field $\xi$. In [36], Chow et al. studied the divergence of the Lie derivative such that

$$
\operatorname{div}\left(\mathscr{L}_{\xi} g\right)=(\Delta+S)(\xi)+d(\operatorname{div}(\xi))
$$

where $\Delta$ represents the Laplace-Hodge operator with respect to the metric $g$ and $S$ is the Ricci curvature tensor field. Now, consider the equation

$$
\mathscr{L}_{\xi} g+2 \alpha S+(2 \Omega-\beta R) g+2 \mu \eta \otimes \eta=0 .
$$

Taking trace of equation (49), we have

$$
\operatorname{div}(\xi)+(\alpha-4 \beta) R+4 \Omega+\mu|\xi|^{2}=0
$$

where $R$ is scalar curvature. By direct calculation, we obtain

$$
\operatorname{div}(\eta \otimes \eta)=\operatorname{div}(\xi) \eta+\nabla_{\xi} \eta
$$

By taking the divergence of (49) and using (48), we obtain

$$
\operatorname{div}\left(\mathscr{L}_{\xi} g\right)+(\alpha-4 \beta) d(R)+2 \mu\left[\operatorname{div}(\xi) \eta+\nabla_{\xi} \eta\right]=0
$$

For Schrödinger-Ricci solution, we say that a 1 -form $\chi$ is a solution of the Schrödinger-Ricci equation if

$$
(\Delta+S)(\chi)+d(\operatorname{div}(\chi))=0 .
$$

Hence, we have next results.

Theorem 22. Let $(g, \xi, \Omega, \mu, \alpha, \beta)$ is an $\eta$-Ricci-Yamabe soliton on an imperfect fluid GRW spacetime $\left(M^{4}, g\right)$ with $\eta$ the $g$-dual of the vector field $\xi$. Then, $\eta$ is the solution of the Schrödinger-Ricci equation if and only if

$$
d(\sigma-3 p)=\frac{2 \mu}{(\alpha-4 \beta)}\left\{[4(\Omega+\lambda)-\mu-k(3 p-\sigma+J)] \eta-\nabla_{\xi} \eta\right\} .
$$

Proof. Using equations (49), (50), (51), and (31) and the fact that $2 \operatorname{div}(S)=(\alpha-4 \beta) d(R)$, it follows that $\eta$ is a solution of the Schrödinger-Ricci equation if and only if (52) holds. $\square$

Theorem 23. Let $(g, \xi, \Omega, \mu, \alpha, 0)$ is the $\eta$-Ricci soliton on an imperfect fluid GRW spacetime $\left(M^{4}, g\right)$ with $\eta$ the $g$-dual of the vector field $\xi$. Then, $\eta$ is a solution of the SchrödingerRicci equation if and only if

$$
d(\sigma-3 p)=\frac{2 \mu}{\alpha}\left\{[4(\Omega+\lambda)-\mu-k(3 p-\sigma+J)] \eta-\nabla_{\xi} \eta\right\} .
$$

Theorem 24. Let $(g, \xi, \Omega, \mu, 0, \beta)$ is the $\eta$-Yamabe soliton on an imperfect fluid GRW spacetime $\left(M^{4}, g\right)$ with $\eta$ the $g$-dual of the vector field $\xi$. Then, $\eta$ is a solution of the SchrödingerRicci equation if and only if

$$
d(\sigma-3 p)=-\frac{\mu}{2 \beta}\left\{[4(\Omega+\lambda)-\mu-k(3 p-\sigma+J)] \eta-\nabla_{\xi} \eta\right\} .
$$

Furthermore, if $J=0$ on the perfect fluid GRW spacetime, then the coming Corollary is stated as follows:

Corollary 25. Suppose $(g, \xi, \Omega, \mu, \alpha, \beta)$ is the $\eta$-Ricci-Yamabe soliton on a perfect fluid GRW spacetime $\left(M^{4}, g\right)$ with $\eta$ the $g$-dual of the vector field $\xi$. Then, $\eta$ is a solution of the Schrödinger-Ricci equation if and only if

$d(\sigma-3 p)=\frac{2 \mu}{(\alpha-4 \beta)}\left\{[4(\Omega+\lambda)-\mu-k(3 p-\sigma+J)] \eta-\nabla_{\xi} \eta\right\}$.

By using Theorems (23) and (24) for particular value of $\alpha$ and $\beta$, similarly we can obtain the results for a perfect fluid GRW spacetime. 
For Schrödinger-Ricci harmonic forms, we say that a 1 -form $\chi$ is a Schrödinger-Ricci harmonic form if

$$
(\Delta+S)(\chi)=0
$$

Furthermore, if $\sigma=3 p$, then the fluid is a radiation fluid if and only if $\mu=0$, which yields the Ricci-Yamabe soliton

$$
\frac{1}{(\alpha-4 \beta)}[4(\Omega+\lambda)-\mu-k(3 p-\sigma+J)] \eta=\nabla_{\xi} \eta
$$

which implies that $\mu=4(\Omega+\lambda)-k(3 p-\sigma+J)$. Hence, we introduce the next results.

Theorem 26. Suppose $(g, \xi, \Omega, \mu, \alpha, \beta)$ is an $\eta$-Ricci-Yamabe soliton on an imperfect fluid GRW spacetime $\left(M^{4}, g\right)$ with $\eta$ the $g$-dual of the vector field $\xi$. Then, $\eta$ is a solution of the Schrödinger-Ricci harmonic form if and only if $\mu=0$, which yields Ricci-Yamabe soliton or

$$
\frac{1}{(\alpha-4 \beta)}[4(\Omega+\lambda)-\mu-k(3 p-\sigma+J)] \eta=\nabla_{\xi} \eta
$$

which implies that $\mu=4(\Omega+\lambda)-k(3 p-\sigma+J)$.

Theorem 27. Let $(g, \xi, \Omega, \mu, 0, \beta)$ is an $\eta$-Yamabe soliton on an imperfect fluid GRW spacetime $\left(M^{4}, g\right)$ with $\eta$ the $g$-dual of the vector field $\xi$. Then, $\eta$ is a solution of the SchrödingerRicci harmonic form if and only if $\mu=0$, which yields Yamabe soliton or

$$
\frac{1}{-4 \beta}[4(\Omega+\lambda)-\mu-k(3 p-\sigma+J)] \eta=\nabla_{\xi} \eta,
$$

which implies that $\mu=4(\Omega+\lambda)-k(3 p-\sigma+J)$.

Remark 28. For $J=0$ and particular value of $\alpha$ and $\beta$ in the Theorems 26 and 27, we can obtain the Schrödinger-Ricci harmonic form for a perfect fluid GRW spacetime.

\section{Some Applications}

Equations (3)-(5) describe the deformations of a Riemannian metric $g_{i j}$ with time $t$. The deformation is driven by Ricci curvature, so that the part of the manifold with greater Ricci curvature will undergo greater deformation. However, the fix points can be computed if the flows are the Ricci flat manifolds Ric $=0$. As a result of Perelman's significant work on Ricci-Yamabe (Ricci-Yamabe solitons) [37], the geometrization of these flows has experienced tremendous growth. It is better to see some following physical applications. In fact, the Ricci-Yamabe soliton is used to understand the idea of kinematics and thermodynamics in general relativity [28, 38]. Ricci-Yamabe solitons are focused because their curvatures keep the self-similarity (self-similar solution).
(1) The spaces are stable or not under geometric flow; this would have application to tachyon condensation in string theory [2]

(2) We can explore Ricci-Yamabe soliton on the manifolds with boundary; in this context, there are applications to black hole and thermodynamics as well as it has some relevance to certain formulations of quasilocal mass $[38,39]$

(3) Laplace-Poisson equation follows the principal of relativity; it describes gravitational field. The azimuthally symmetric theory of gravitation (ASTGmodel) and Magneto-Hydro-Dynamic (MHD) [39] modeling of molecular clouds are also based on the Laplace-Poisson equation

\section{Conclusions}

In GR, the matter content of the universe is considered to work like an imperfect fluid in the standard cosmological models such as a time oriented 4-dimensional Lorentzian manifold. In this framework, Einstein's equation plays the fundamental role to construct the cosmological models. Relativistic imperfect GRW fluid spacetime [40] models are of considerable interest in several areas of astrophysics [2, 41, 42], plasma physics [3], and nuclear physics [1, 43, 44].

The propagation of fundamental field on black hole spacetime [45], which is a relevant case of black hole spacetime in the presence of plasma. An ideal MHD is the realization that plasma elements connected by magnetic field line at a given time will remain connected by a magnetic field line at any subsequent time, provided that the plasma velocity field remains smooth. This property arises because a plasma that satisfies the Ohm's law moves with a transport velocity that preserves the magnetic connection between plasma elements [46]. Moreover, plasma elements can be cast in a covariant form in a specific foliation spacetime [47]. Thus, Ohm's law

$$
F^{c} T_{a b}=0
$$

where $F^{c}$ is the four fluid velocity vector field and $T_{a b}$ is the electromagnetic filed tensor. The above equation provides a simple and effective constitute relation for large scale and low frequency plasma dynamics [3].

On the other side, geometric flows are most effective tools to describe the geometric structures in relativistic imperfect fluid GRW spacetime (semi-Riemannian geometry) [29]. A special class of solutions on which the metric evolves by dilation and diffeomorphisms plays a crucial role in the study of singularities of the flows, as they appear as possible singularity models $[16,37]$. They are often called soliton solutions (Ricci-Yamabe solitons).

A 4-dimensional imperfect fluid GRW spacetime manifold model is conceding Ricci-Yamabe soliton. RicciYamabe solitons are the natural extension of the Einsteins metric in semi-Riemannian geometry. Therefore, Einstein manifolds arose during the study of exact solution of the Einstein's field equation. We turn up the condition such as 
semiconformally flat imperfect fluid GRW spacetime, semisymmetric, and Ricci semisymmetric imperfect fluid GRW spacetime with Ricci-Yamabe soliton. Finally, we discuss some harmonic significance of imperfect fluid GRW spacetime in terms of $\eta$-Ricci-Yamabe soliton.

\section{Data Availability}

No data were used to support this study.

\section{Conflicts of Interest}

On behalf of all authors, the corresponding author states that there is no conflict of interest.

\section{Acknowledgments}

The first author (A. H. Al-Khaldi) extends their appreciation to the Deanship of Scientific Research at King Khalid University for funding this work through the Research Groups Program under grant number R.G.P2/74/42.

\section{References}

[1] M. A. Canadas-Piedo, M. Gutierrez, and M. Ortega, "Massless particles in generalized Robertson-Walker 4-spacetimes," Annali di Matematica Pura ed Applicata, vol. 194, no. 1, pp. 259-273, 2015.

[2] S. W. Hawking and G. F. Ellis, $R$ the Large Scale Struture of Spacetime, Cambridge Monographs on Mathematical Physics Vol. 1, Cambridge University Press, London, 1973.

[3] D. Biskamp, Reconnection in Plasma, Cambridge University Press, Cambridge, London, 2000.

[4] L. Alias, A. Romero, and M. Sanchez, "Uniqueness of complete spacelike hypersurfaces of constant mean curvature in generalized Robertson-Walker spacetime," General Relativity and Gravitation, vol. 30, pp. 915-932, 1995.

[5] C. A. Mantica and L. G. Molinari, "Generalized RobertsonWalker spacetimes, a survey," International Journal of Geometric Methods in Modern Physics, vol. 14, no. 3, article 102502, 2017.

[6] A. A. Shaikh, "On pseudo quasi-Einstein manifolds," Periodica Mathematica Hungarica, vol. 59, no. 2, pp. 119-146, 2009.

[7] M. Sanchez, "On the geometry of generalized RobertsonWalker spacetime geodesics," General Relativity and Gravitation, vol. 27, pp. 71-84, 1998.

[8] B. O'Neill, Semi-Riemannian Geometry with Applications to Relativity, Academic Press, New York, 1983.

[9] M. Ali and Z. Ahsan, "Ricci solitons and symmetries of space time manifold of general relativity," Journal of Advanced Research on Classical and Modern Geometries, vol. 1, no. 2, pp. 75-84, 2014.

[10] A. M. Blaga, "Solitons and geometrical structures in a perfect fluid spacetime," The Rocky Mountain Journal of Mathematics, vol. 50, no. 1, 2020.

[11] K. H. Venkatesha, "Ricci solitons and geometrical structure in a perfect fluid spacetime with torse-forming vector filed," Afrika Mathematika, vol. 30, no. 5, pp. 725-736, 2019.

[12] Z. Ahsan and S. A. Siddiqui, "Concircular curvature tensor and fluid spacetimes," International Journal of Theoretical Physics, vol. 48, no. 11, pp. 3202-3212, 2009.
[13] M. C. Chaki and S. Ray, "Spacetimes with covariant constant energy momentum tensor," International Journal of Theoretical Physics, vol. 35, no. 5, pp. 1027-1032, 1996.

[14] M. D. Siddiqi and S. A. Siddqui, "Conformal Ricci soliton and geometrical structure in a perfect fluid spacetime," International Journal of Geometric Methods in Modern Physics, vol. 17, no. 6, article 2050083, 2020.

[15] S. Güler and M. Crasmareanu, "Ricci-Yamabe maps for Riemannian flows and their volume variation and volume entropy," Turkish Journal of Mathematics, vol. 43, no. 5, pp. 2631-2641, 2019.

[16] R. S. Hamilton, "The Ricci flow on surfaces," in Mathematics and General Relativity, pp. 237-262, American Mathematical Society, 1988.

[17] G. Catino and L. Mazzieri, "Gradient Einstein solitons," Nonlinear Analysis, vol. 132, pp. 66-94, 2016.

[18] J. T. Cho and M. Kimura, "Ricci solitons and real hypersurfaces in a complex space form," Tohoku Mathematical Journal, vol. 61, no. 2, pp. 205-212, 2009.

[19] A. M. Blaga, "On gradient $\eta$-Einstein solitons," Kragujevac Journal of Mathematics, vol. 42, no. 2, pp. 229-237, 2018.

[20] A. M. Blaga, "Harmonic aspects in an $\eta$-Ricci soliton," International Electronic Journal of Geometry, vol. 13, no. 1, pp. 41-49, 2020.

[21] M. Crasmareanu, "A new approach to gradient Ricci solitons and generalizations," Univerzitet $u$ Nišu, vol. 32, no. 9, pp. 3337-3346, 2018.

[22] B. Y. Chen, "A simple characterization of generalized Robertson-Walker spacetimes," General Relativity and Gravitation, vol. 46, no. 12, p. 1833, 2014.

[23] S. Güler, "On a class of gradient almost Ricci solitons," Bulletin of the Malaysian Mathematical Sciences Society, vol. 43, no. 5, pp. 3635-3650, 2020.

[24] M. D. Siddiqi, "Ricci $\rho$-soliton and geometrical structure in a dust fluid and viscous fluid sapcetime," Bulgarian Journal of Physics, vol. 46, pp. 163-173, 2019.

[25] A. E. Fischer, "An introduction to conformal Ricci flow," Classical and Quantum Gravity, vol. 21, no. 3, pp. S171-S218, 2004.

[26] K. Duggal, "Almost Ricci solitons and physical applications," International Electronic Journal of Geometry V, vol. 10, no. 2, pp. 1-10, 2017.

[27] H. Stephani, General Relativity-An Introduction to the Theory of Gravitational Field, Cambridge University Press, Cambridge, 1982.

[28] W. S. Hipolito-Ricaldi, H. E. S. Velten, and W. Zimdahl, "Viscous dark fluid universe," Physical Review D, vol. 82, no. 6, article 063507, 2010.

[29] C. A. Mantica and L. G. Molinari, "A condition for a perfect fluid spacetime to be a generalized Robertson-Walker spacetimes, a survey," Journal of Mathematical Physics, vol. 57, no. 2, article 049901, 2016.

[30] K. Yano, "On torse forming direction in a Riemannian space," Proceedings of the Imperial Academy, vol. 20, no. 6, pp. 340345, 1994.

[31] M. Novello and M. J. Reboucas, "The stability of a rotating universe," The Astrophysical Journal, vol. 225, pp. 719-724, 1978.

[32] S. K. Chaubey, "Generalized Robertson-Walker space-time with $\mathrm{W}_{1}$-curvature tensor," Journal of Physics A, vol. 10, no. 2, article 1000303, 2019. 
[33] J. Kim, “A type of conformal curvature tensor," Far East Journal of Mathematical Sciences, vol. 99, no. 1, pp. 61-74, 2015.

[34] J. Kim, "On pseudo semiconformally symmetric manifolds," Bulletin of the Korean Mathematical Society, vol. 54, no. 1, pp. 177-186, 2017.

[35] K. Yano, Integral Formulas in Riemannian Geometry, Marcel Dekker, New York, 1970.

[36] B. Chow, S. C. Chu, D. Glickenstein et al., "The Ricci flow: techniques and applications," in Part I: Geometric Aspects 135, AMS, 2007.

[37] G. Perelman, "The entrophy formula for the Ricci flow and its geometric applications," 2002, http://arxiv.org/abs/math/ 0211159 .

[38] C. Eckart, "The thermodynamics of irreversible processes III. Relativistic theory of the simple fluid," Physics Review, vol. 58, no. 10, pp. 919-924, 1940.

[39] P. Davidson, An Introduction to Magnetohydrodynamics, Cambridge University Press, Cambridge, UK, 2nd edition, 2017.

[40] R. Maartens, "Causal thermodynamics in relativity," in Lectures given at the Hanno Rund Workshop on Relativity and Thermodynamics, Natal University, South Africa, 1996, http://arxiv.org/abs/9609119.

[41] R. R. Caldwell, "A phantom menace? Cosmological consequences of a dark energy component with super-negative equation of state," Physics Letters B, vol. 545, no. 1-2, pp. 23$29,2002$.

[42] P. H. Chavanis, "Chavanis cosmology with a stiff matter era," Physical Review D, vol. 92, no. 10, article 103004, 2015.

[43] M. Barros, M. Caballero, and M. Ortega, "Massless particles in warped three spaces," International Journal of Modern Physics A, vol. 21, no. 3, pp. 461-473, 2006.

[44] M. S. Plyushchay, "The model of the relativistic particle with torsion," Nuclear Physics B, vol. 362, no. 1-2, pp. 54-72, 1991.

[45] W. A. Newcomb, "Motion of magnetic lines of force," Annals of Physics, vol. 3, no. 4, pp. 347-385, 1958.

[46] G. Hornig, "The covariant transport of electromagnetic fields and its relation to magnetohydrodynamics," Physics of Plasmas, vol. 4, no. 3, pp. 646-654, 1997.

[47] F. A. Asenjo and L. Cosmisso, "Magnetic connection in curved spacetime," Physical Review D, vol. 96, article 122004, 2017. 